\title{
Effect of High Osmotic Pressure Milk Formula on Renal Calcium Oxalate Crystallization Induced by Calcium Oxalate
}

\author{
Meihong $\mathrm{Xu}^{1}$, Liren $\mathrm{Wei}^{1}$, Zhiyong $\mathrm{Dai}^{2}$, Yanchun Zhang ${ }^{2}$, Yong $\mathrm{Li}^{1}$, Junbo Wang ${ }^{1, *}$ \\ ${ }^{1}$ Department of Nutrition and Food Hygiene, School of Public Health, Peking University, Beijing, PR China \\ ${ }^{2}$ R\&D center of Ausnutria Hyproca Dairy Group, Changsha, PR China \\ *Corresponding author: mrwang4j@163.com
}

Received November 25, 2014; Revised January 22, 2015; Accepted February 03, 2015

\begin{abstract}
To study the effect of high osmotic pressure milk formula on renal calcium oxalate crystallization induced by calcium oxalate. 40 SD rats were randomly divided into four groups: NC, MC, LOM and HOM group. For model preparation, MC, LOM and HOM were treated with 1\% Ethanediol in the water combined with $2 \%$ ammonium chloride (2ml/day) intragastrically administration. The rats in LOM and HOM were intragastrically given LOM or HOM $(40 \mathrm{~g} / 100 \mathrm{ml}, 2 \mathrm{ml} / 100 \mathrm{~g} * \mathrm{bw})$ twice/day, whereas the NC and MC group received an equal volume of distilled water. Combined application of ethylene glycol and ammonium chloride caused renal injury of rats, and induced calcium oxalate calculus in kidney. During experimental period, the increase of body weight of the rats treated with HOM was inhibited significantly; the amount of 24h urination, serum levels of $\mathrm{Cr}$ and UA, ratio of the kidney to body weight were significantly higher than those in MC group; the amount of 24h urination, $\mathrm{Cr}$, and score of calcium oxalate crystal were significantly higher than those in LOM group. HOM has an obvious promoting effect on the formation of renal calcium oxalate crystallization induced by ethylene glycol combined with ammonium chloride.
\end{abstract}

Keywords: infant formula, osmotic pressure, renal calcium oxalate crystallization

Cite This Article: Meihong Xu, Liren Wei, Zhiyong Dai, Yanchun Zhang, Yong Li, and Junbo Wang, "Effect of High Osmotic Pressure Milk Formula on Renal Calcium Oxalate Crystallization Induced by Calcium Oxalate.” Journal of Food and Nutrition Research, vol. 3, no. 2 (2015): 94-98. doi: 10.12691/jfnr-3-2-3.

\section{Introduction}

Recently, the trend of urinary tract calculi onset, in infants and children, is obvious rising. And the location is mostly (about 80\%) at kidney [1,2]. Urinary calculus can be divided into two categories, primary and secondary. The etiology of secondary urinary calculus was complex, including individual factors and environmental factors. Based on the existing research, there were still no consistent conclusion on the cause of kidney calculus. Various factors could associated with it, such as food, climate, occupation and genetic factors.

It has been shown, by Melamine Milk Incident, that the influence of diet cannot be ignored [3]. Formula, as a major food sources for the infants, might be correlated with the formation of renal calculus infants. Especially, the osmotic pressure of milk powder may be affecting the formation of renal calculus through influencing body metabolism. The osmotic pressure of milk is mainly composed of the inorganic solvents. It has been shown that the high osmotic press milk may have an impaction on infant's kidney function [4,5]. However, the study of milk osmotic pressure, as well as the relationship between osmotic pressure and renal calculus, is scarce.
Therefore, in the present paper, it has been discussed the effect of high osmotic pressure milk formula on renal calcium oxalate crystallization induced by calcium oxalate. In order to provide reasonable references for the feeding of infant feeding and the control of renal calculus.

\section{Materials and Methods}

\subsection{Material}

Low osmotic pressure milk powder (LOM) and high osmotic pressure milk powder (HOM) were provided by Ausntria Hyproca Dairy Group BV. (Hunan, China). The HOM was supplemented with $2 \mathrm{~g}$ salt (without crystal water) to $100 \mathrm{~g}$ LOM. The composition of salt was $0.583 \mathrm{~g}$ $\mathrm{CaCl}_{2}, 1.113 \mathrm{~g} \mathrm{k}_{3} \mathrm{PO}_{4}$ and $0.304 \mathrm{~g} \mathrm{NaCl}$. Ethanediol, ammonium chloride and the other chemicals used were all of analytical grade and purchased from Beijing Chemical Co. (Beijing, China).

\subsection{Animals and Experimental Procedure}

A total of 40 male weaned SD rats, weighing 180-220 g, were obtained from the Animal Service of Health Science Center, Peking University and adapted to the vivarium for one week before the treatment began. The conditions 
consisted of a filter-protected air-conditioned room, with a controlled temperature $\left(25 \pm 28^{\circ} \mathrm{C}\right)$, relative humidity (60 $\pm 5 \%$ ) and $12 \mathrm{~h}$ light/dark cycles (light on between 07:30 and 19:30 h). The animal treatment and maintenance were conducted in complete accordance with the Principle of Laboratory Animal Care (NIH Publication No. 85-23, revised 1985) and the guidelines of the Peking University Animal Research Committee.

Intervention started after the one week adaption. The animals were randomly assigned to 4 groups, including a normal control group (defined as NC group), a model control group (defined as MC group), a low osmotic pressure milk formula group (defined as LOM group), an high osmotic pressure milk formula group (defined as HOM group). All of the rats were fed with a model AIN93G rodent diet (Vital River Limited Company, Beijing, China). For the model preparation, the rats, in MC, LOM and HOM were intragastrically $2 \%$ ammonium chloride ( $2 \mathrm{ml} /$ day) combined with drank the special water which were added with 1\% Ethanediol. Meanwhile, the rats, in LOM and HOM group, were intragastrically administered LOM or HOM $(40 \mathrm{~g} / 100 \mathrm{ml}, 2 \mathrm{ml} / 100 \mathrm{~g} * \mathrm{bw})$ twice/day, whereas the NC and MC group received an equal volume of distilled water.

Animal were observed daily and weekly for adverse clinical sign. A detailed physical examination was conducted at least once during the pretreatment period, once weekly during the test substance administration and on the day of necropsy. Body weight, food consumption were recorded weekly. Water consumption were recorded every two days form the 4th week. On the 45th day, the urine was collected in 24 hours. The volume of urine was recorded. The study lasted for 7 weeks (49 days). Day 49, at $16 \mathrm{~h}$ after fasting, the rats were anesthetized by $\mathrm{CO}_{2}$ inhalation and then sacrificed.

\subsection{Osmotic Pressure of Milk Formula Determine}

The samples of LOM and HOM were rushed into solution by distilled water, according to the standard method (14g/100ml). Then, detect the osmotic pressure of both, using osmotic pressure molar concentration meter (STY- type 2, Beijing, China)

\subsection{Biochemical Assays}

Blood samples were collected form femoral artery for serum chemistry from rats immediately prior to the scheduled necropsy on day 49. The serum was separated by centrifugation at $3000 \mathrm{rpm}$ for $10 \mathrm{~min}$. The levels of creatinine $(\mathrm{Cr})$ and blood urea nitrogen (BUN) in the serum were detected using an automatic biochemistry analyzer (Hitachi 7020, Tokyo, Japan).

\subsection{Uric Assays}

The levels of Urinary calcium in the uric were detected using an atomic absorption spectrophotometry analyzer (East\&West AA7000M, Beijing, China).

\subsection{Histological Study}

Portions of the kidney for histopathological examination were also obtained. The surrounding tissue of renal hilus were preserved in neutral buffered formalin and were processed for paraffin embedding, following the standard microtechnique. Four to five micron sections of kidneys stained with haematoxylin and eosin. And then, the changes of inflammatory were observed under microscope (Nikon, Y-FL light microscope, Japan).

The degree of calcium oxalate crystallization was reviewed as following standards:

0: no crystallization;

1: crystallization bright spot in scattered;

2: a wide range of piles or confined crystallization;

3: piles of crystal powder without connection;

4: crystal piles and connected to each other;

5: widely piles of crystallization, connection piece.

\subsection{Statistical Analysis}

Statistical analyses were performed using SPSS software (version 19.0, SPSS Inc., Chicago, IL, USA). Variances in the measurement data were checked for homogeneity by Bartlett's test. When the data were homogeneous, the one-way analysis of variance test and LSD methods were used. Tamhane's T2 test is used after data are transformed to analyze data among multiple groups if variances are unequal. All reported $\mathrm{P}$ values were two-sided. A value of $\mathrm{P}<0.05$ was considered significant.

\section{Results}

\subsection{General Information}

There was no remarkable difference in general condition and behavior among the NC, MC, LOM and HOM rats.

Table 1. Weight dynamics in rats with different level of osmotic pressure milk formula

\begin{tabular}{|c|c|c|c|c|c|c|c|c|c|}
\hline \multirow{2}{*}{ Group } & \multirow{2}{*}{$\mathrm{N}$} & \multicolumn{8}{|c|}{ Body weight (g) } \\
\hline & & Baseline & $1^{\text {st }}$ week & $2^{\text {nd }}$ week & $3^{\text {rd }}$ week & $4^{\text {th }}$ week & $5^{\text {th }}$ week & $6^{\text {th }}$ week & $7^{\text {th }}$ week \\
\hline $\mathrm{NC}$ & 10 & $50.8 \pm 1.8$ & $93.4 \pm 4.9$ & $152.7 \pm 9.2$ & $214.2 \pm 10.0$ & $270.8 \pm 12.5$ & $332.2 \pm 10.4$ & $362.0 \pm 19.5$ & $376.8 \pm 20.5$ \\
\hline $\mathrm{MC}$ & 10 & $50.0 \pm 2.1$ & $93.4 \pm 3.1$ & $152.6 \pm 6.0$ & $207.1 \pm 10.3$ & $265.4 \pm 13.2$ & $318.0 \pm 20.2$ & $350.1 \pm 18.1$ & $354.4 \pm 27.2$ \\
\hline LOM & 10 & $50.0 \pm 3.0$ & $91.2 \pm 5.4$ & $142.0 \pm 11.6$ & $198.7 \pm 16.4$ & $248.6 \pm 21.0$ & $297.9 \pm 21.6^{\mathrm{a}}$ & $344.0 \pm 21.2$ & $360.7 \pm 16.4$ \\
\hline HOM & 10 & $50.7 \pm 2.1$ & $89.5 \pm 3.3$ & $138.2 \pm 4.7^{\mathrm{ab}}$ & $187.4 \pm 15.7^{\mathrm{ab}}$ & $238.8 \pm 21.3^{\mathrm{ab}}$ & $278.6 \pm 26.0^{\mathrm{ab}}$ & $330.9 \pm 29.1^{\mathrm{a}}$ & $306.2 \pm 22.1^{a b c}$ \\
\hline
\end{tabular}

Data are mean \pm SD values. $\mathrm{N}=10$.

Data were analyzed by one-way analysis of variance. Multiple comparison by Dunnett's t test was used to evaluate the difference.

a. Statistical significance: $\mathrm{p}<0.05$, compared with NC group.

b. Statistical significance: $\mathrm{p}<0.05$, compared with MC group.

c. Statistical significance: $\mathrm{p}<0.05$, compared with LOM group.

NC group, normal control group; MC group, model control group; LOM group, low osmotic pressure milk formula; HOM group, high osmotic pressure milk formula. 
As shown in Table 1, the body weight of HOM group was significantly lighter than $\mathrm{NC}$ and $\mathrm{MC}$ group, form week 2 to the end of experiment $(p<0.05)$. However, there were no remarkable differences between LOM and NC group ( $>0.05)$, with the exception of week $5(p<0.05)$.

\subsection{Osmotic Pressure of Milk Formula}

At the concentration of $14 \mathrm{~g} / 100 \mathrm{ml}$, the osmotic pressure of LOM and HOM was $319.72 \pm 4.22$ $\mathrm{mOsmol} / \mathrm{kg}$ and $490.74 \pm 0.43 \mathrm{mOsmol} / \mathrm{kg}$, respectively.

\subsection{Water Consumption, Urination and Urinary Calcium}

As shown in Figure 1, there were no significant differences in water consumption among the four groups $(p>0.05)$. The urination was remarkable increased in HOM group than NC, MC and LOM group $(\mathrm{p}<0.05)$. Conversely, the urinary calcium was increased in MC, LOM and HOM group $(\mathrm{p}<0.05)$. However, there were no differences among the HOM group, LOM and MC group $(\mathrm{p}>0.05)$.

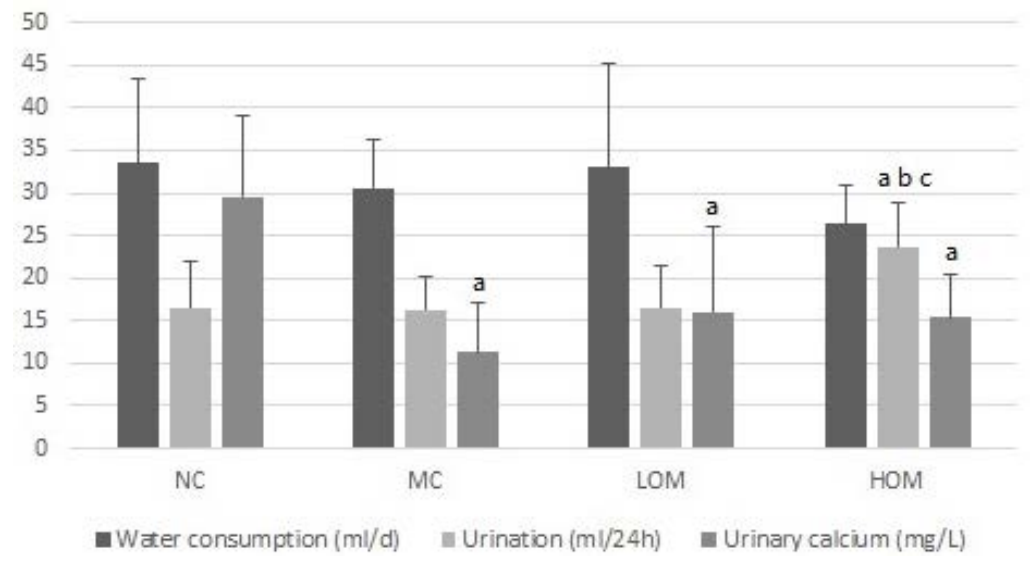

Figure 1. Water consumption, urination and urinary calcium in rats with different formula

Data were analyzed by one-way analysis of variance. Multiple comparison by Dunnett's t test was used to evaluate the difference.

a. Statistical significance: $\mathrm{p}<0.05$, compared with NC group.

b. Statistical significance: $\mathrm{p}<0.05$, compared with MC group.

c. Statistical significance: $\mathrm{p}<0.05$, compared with LOM group.

NC group, normal control group; MC group, model control group; LOM group, low osmotic pressure milk formula; HOM group, high osmotic pressure milk formula.

\subsection{Serum Biochemical Indicators}

Analysis biochemical parameters, the changes were shown as follows (Table 2). In comparison with NC group, the level of Ca was increased in MC group $(\mathrm{p}<0.05)$; similarly, the level of $\mathrm{Ca}$, in HOM group, was higher than that in NC, MC and LOM group ( $<<0.05)$.

After model preparation by Ethanediol and ammonium chloride, the level of BUN and $\mathrm{Cr}$ was increased, and there were significant differences, compared to NC group $(\mathrm{p}<0.05)$. The level of BUN and $\mathrm{Cr}$, in HOM group, was higher than MC group ( $\mathrm{p}<0.05)$. The level of $\mathrm{Cr}$ in HOM group was also higher than that in LOM group $(\mathrm{p}<0.05)$. There were no remarkable differences between MC and LOM group ( $\mathrm{p}>0.05)$.

Compared to MC group, the level of UA was significantly higher in NC group and lower in LOM and HOM group $(p<0.05)$. However, there were no remarkable differences between LOM and HOM group ( $p>0.05)$.

Table 2. Ca, BUN, Cr and UA levels in rats with different level of osmotic pressure milk formula

\begin{tabular}{|c|c|c|c|c|c|}
\hline Group & $\mathrm{N}$ & $\begin{array}{l}\mathrm{Ca} \\
(\mathrm{mmol} / \mathrm{L})\end{array}$ & $\begin{array}{l}\mathrm{BUN} \\
(\mathrm{mmol} / \mathrm{L})\end{array}$ & $\begin{array}{l}\mathrm{Cr} \\
(\mathrm{mol} / \mathrm{L})\end{array}$ & $\begin{array}{l}\text { UA } \\
(\mathrm{mol} / \mathrm{L})\end{array}$ \\
\hline NC & 10 & $3.04 \pm 0.11$ & $5.84 \pm 0.68$ & $6.26 \pm 2.85$ & $134.61 \pm 15.02$ \\
\hline MC & 10 & $3.32 \pm 0.05^{\mathrm{a}}$ & $8.25 \pm 1.30^{a}$ & $15.23 \pm 1.98^{\mathrm{a}}$ & $160.60 \pm 19.04^{\mathrm{a}}$ \\
\hline LOM & 10 & $2.96 \pm 0.11^{b}$ & $9.25 \pm 2.38^{a}$ & $18.71 \pm 2.73^{\mathrm{a}}$ & $139.57 \pm 11.97^{b}$ \\
\hline HOM & 10 & $3.18 \pm 0.06^{a b c}$ & $10.66 \pm 1.92^{\mathrm{ab}}$ & $28.89 \pm 8.75^{\mathrm{abc}}$ & $136.28 \pm 11.42^{b}$ \\
\hline
\end{tabular}

Data are mean \pm SD values. $\mathrm{N}=10$.

Data were analyzed by one-way analysis of variance. Multiple comparison by Dunnett's t test was used to evaluate the difference.

a. Statistical significance: $\mathrm{p}<0.05$, compared with NC group.

b. Statistical significance: $\mathrm{p}<0.05$, compared with MC group

c. Statistical significance: $\mathrm{p}<0.05$, compared with LOM group.

d. Ca, blood calcium; BUN, blood urea nitrogen; Cr, serum creatinine; UA, uric acid; NC group, normal control group; MC group, model control group; LOM group, low osmotic pressure milk formula; HOM group, high osmotic pressure milk formula.

\subsection{Histological analysis}

After model preparation, the weight of kidney and kidney to body weight was significantly higher than NC group $(\mathrm{p}<0.05)$. Compared to MC group, the weight of kidney and kidney, in LOM and HOM group, was significantly higher than that in MC group $(\mathrm{p}<0.05)$.
However, there were no remarkable differences between LOM and HOM group ( $\mathrm{p}>0.05$ ).

For the pathological part, results were as follows (shown in Figure 2). In NC group, it did not shown significant anomalies. Renal capsule was complete, which was composed of dense connective tissue; the multiple glomeruli was scattered in renal cortex, and its structure 
was clear; red dye was not observed in Bowman's capsule; there was a clear structure in renal tubular, around the glomerulus; and the structure of renal tubular, in the renal medulla, is integrity. Nevertheless, in MC group, kidney tissue was shown serious inflammation. Under the microscopy, there was a large number of edema in renal tissue; renal tubular became thickened; glomerulus was atrophy; convoluted tubules were arranged in disorder; and inflammatory cells was found in pelvic. In the LOM and HOM group, there were visible tissue edema, but it had less inflammatory cells in the renal pelvis.
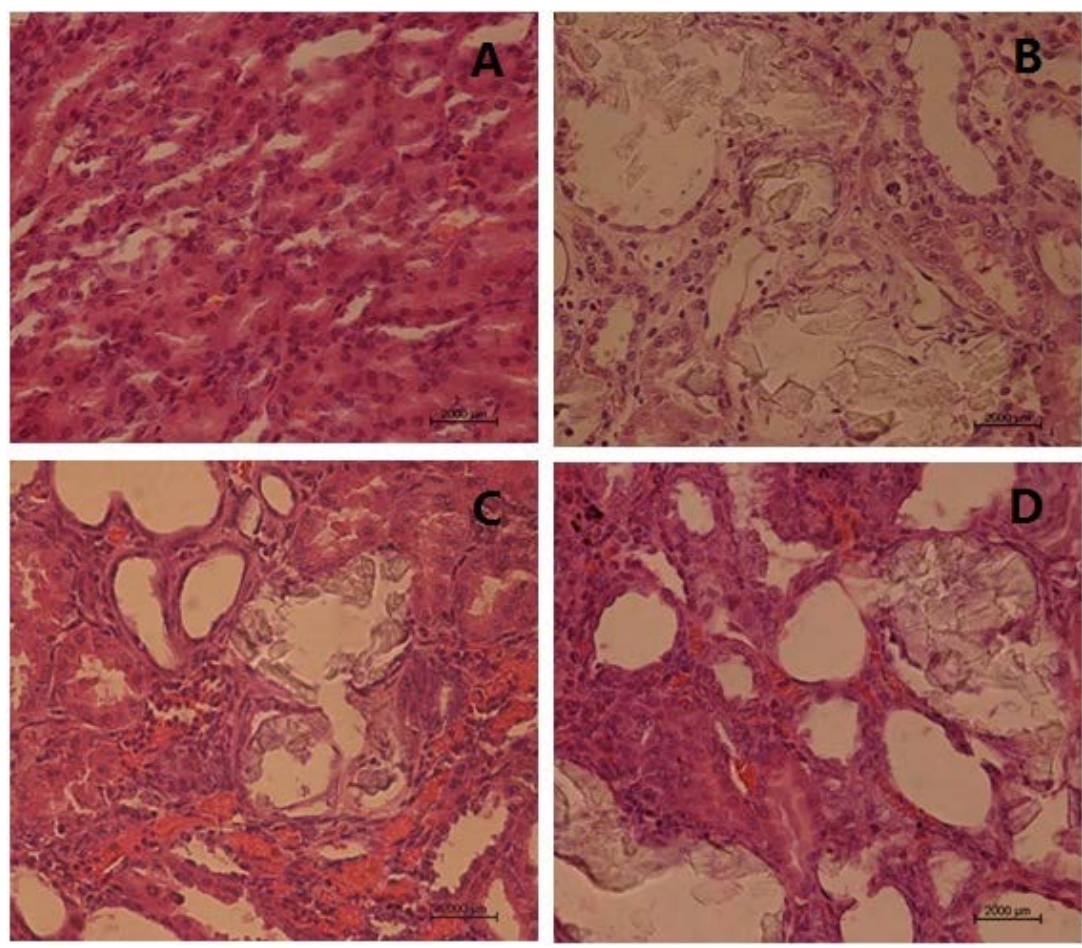

Figure 2. A-D. Representative micrograph of haematoxylin and eosin-stained section of kidney treated with different formula (A. NC group; B. MC group; C, LOM group; D, HOM group; Magnification, 10×40)

For the model preparation groups, the scores were higher than NC group in left kidney, right kidney and total $(\mathrm{p}<0.05)$. There were remarkable differences, in right kidney and total score, between HOM and LOM group $(\mathrm{p}<0.05)$ (Table 3).

Table 3. Score of calcium oxalate crystals in rat's kidney with different level of osmotic pressure milk formula

\begin{tabular}{ccccc}
\hline \multirow{2}{*}{ Group } & $\mathrm{N}$ & \multicolumn{3}{c}{ Score } \\
\cline { 3 - 5 } & & Left kidney & Right kidney & Total \\
\hline NC & 10 & $0.00 \pm 0.00$ & $0.00 \pm 0.00$ & $0.00 \pm 0.00$ \\
MC & 10 & $1.90 \pm 0.74^{\text {a }}$ & $2.40 \pm 0.97^{\text {a }}$ & $4.30 \pm 1.49^{\text {a }}$ \\
LOM & 10 & $1.80 \pm 1.14^{\text {a }}$ & $1.70 \pm 1.06^{\text {a }}$ & $3.50 \pm 1.65^{\text {a }}$ \\
HOM & 10 & $2.40 \pm 1.17^{\text {a }}$ & $2.50 \pm 0.97^{\text {ac }}$ & $4.90 \pm 1.79^{\text {ac }}$ \\
\hline
\end{tabular}

Data are mean \pm SD values. $\mathrm{N}=10$.

Data were analyzed by one-way analysis of variance. Multiple comparison by Dunnett's t test was used to evaluate the difference.

\section{Discussion}

Breast milk is the most ideal natural food for infants. How to rational choose the infant formula is the key of infant's growth and disease control. The osmotic pressure of the emulsion is reflected the amount of a chemical parameters of the solute, consist of crystal osmotic pressure and colloid osmotic pressure. The priority of osmotic pressure in milk is crystal osmotic pressure. The osmotic pressure of emulsion of are mainly depended on electrolytes (sodium, potassium, etc.) and the concentration of sugar. According to research, hypertonic emulsion (osmotic pressure value is equal or greater than 300 mosm $/ \mathrm{Kg} \mathrm{H}_{2} \mathrm{O}$ ) not only can lead to high blood sodium and high nitrogen disease. It is also an important factor of infant necrotizing induced colitis [6] and it has bad effect on pediatric kidney [7].

At present, the research on the relationship between solution osmotic pressure and kidney stones is scarce. It has been reported that High osmotic pressure of milk is one of the leading causes of infant kidney stone formation, and it is related with the composition in milk, such as xanthine oxidase, nucleotide, protein content and composition, nonprotein nitrogen and melamine, ash content and monovalent ions and so on.

The function of glomerulus has yet to mature in the babies. It will gradually close to the level of adults, when they were reaching 1 to 2 years old. Because of that, the metabolic function of infant is insufficient. High osmotic solution may cause disorder of infant fluid balance, which could affect the normal function of kidney. Studies had shown that artificial high osmotic pressure emulsion and high osmotic pressure breast milk can give rise to the damage of infant kidney $[4,5,8]$. In the current study, HOM has the effect on renal calcium oxalate crystallization induced by calcium oxalate.

As present study, we supplied $\mathrm{CaCl}_{2}, \mathrm{k}_{3} \mathrm{PO}_{4}$ and $\mathrm{NaCl}$ into normal milk formula, in order to make high osmotic pressure milk formula. After the analysis, the osmotic pressure of LOM and HOM was $319.72 \pm 4.22$ $\mathrm{mOsmol} / \mathrm{kg}$ and $490.74 \pm 0.43 \mathrm{mOsmol} / \mathrm{kg}$, respectively. There were remarkable differences between LOM and 
HOM. According to the medicine on the classification of the osmotic pressure, it belongs to the hypertonic solution.

We treated rats, in MC, LOM and HOM group, with the combined application of ethylene glycol and ammonium chloride, in order to induce calcium oxalate calculus in kidney. Rats in MC group were growth retardation. The level of blood Ca was increased, oppositely, urinary Ca was decreased. That was indicated that calcium may accumulate in the form of crystal in renal tissue. Moreover, there were significant damage in kidney, as well as the levels of UA, Cr and BUN were significantly changed. According to the pathology results, the kidney tissue of NC rats had serious inflammatory response, and they were presenting a lot of calcium oxalate crystal. Similarly, during experimental period, the increase of body weight of the rats treated with HOM was inhibited significantly; the amount of 24h urination, serum levels of Cr and UA, ratio of the kidney to body weight were significantly higher than those in MC group; the amount of 24h urination, Cr, and score of calcium oxalate crystal were significantly higher than those in LOM group. It is indicated that HOM has an obvious promoting effect on the formation of renal calcium oxalate crystallization induced by ethylene glycol combined with ammonium chloride.

HOM used in this study was only added inorganic salt in the formula, which mean the other ingredients of HOM was the same as that in LOM. Based on that, we speculated the reason, why HOM could play a promoted role in stone formation, is the rise of inorganic salt content. There were relationship between the occurrence of urinary calculi and metabolism of the body. It has been reported that ions, such as calcium, phosphorus, magnesium, uric acid, etc., were could influence the formation of kidney calculus through the following pathways: (1) directly involved in or on the crystallization, growth, gathered of calcium salt; Start or inhibit crystallization nucleation or combination with the surface; Change the surface charges, promote or inhibit the growth of crystals and aggregation, or influence the risk of stone formation by affecting calculi formation ion environment. (2) Some elements with renal toxicity could influence the glomerular filtration and renal tubular reabsorption function, so as to change the chemical composition and urine $\mathrm{PH}$, morphology and function of renal tubular epithelial cells. Crystal could adherent to nucleation or damage renal interstitial cells easily, which will prompt renal calcification [9]. Valentini thought that the rise of renal calcium morbidity, in infants and young children, may relate to the excessive intake of salt in processed foods or fast food [10].

Osmotic pressure as a simple indicator, could comprehensively reflect the inorganic salt content of small molecules in milk. Although whether it has a relationship between the inorganic element and infant stone is still unclear, the result of our study could provide a new clues for the further study of etiology and prevention on calculi disease, as well as the references for the rational inorganic salts use in infant formula.

\section{Conclusions}

In present paper, we observed the effect of HOM on renal calcium oxalate crystallization by model preparation. The results indicated that HOM has an obvious promoting effect on the formation of renal calcium oxalate crystallization induced by ethylene glycol combined with ammonium chloride.

\section{Acknowledgement}

The authors gratefully thank Ausnutria Hyproca Dairy Group for providing the samples used in this study.

\section{References}

[1] Novak TE, Lakshmanan Y, Trock BJ, Gearhart JP, Matlaga BR. (2009). Sex prevalence of pediatric kidney stone disease in the United States: an epidemiologic investigation. Urology. 74:104-7

[2] Routh JC, Graham DA, Nelson CP. (2010). Epidemiological trends in pediatric urolithiasis at United States freestanding pediatric hospitals. J Urol. 184: 1100-4.

[3] Ross SS, Masko EM, Abern MR, Allott EH, Routh JC, Wiener JS, Preminger GM, Freedland SJ, Lipkin ME. (2013). The effect of dietary sodium and fructose intake on urine and serum parameters of stone formation in a pediatric mouse model: a pilot study. $\mathrm{J}$ Urol. 190: 1484-9.

[4] Yaohua W, Zhimin D. (2006). The comparison of high osmotic pressure breast milk and osmotic pressure of breastfeeding on infant urinary mAlb, RBP. Public Medical Forum Magazine. 10:316 [article in chinese].

[5] Yaohua W, Zhimin D. (2006). Effect of high osmotic pressure of artificial compound emulsion for feeding babies on renal function: 31 cases of clinical observation. Public Medical Forum Magazine. 10:521 [article in chinese].

[6] Jacqueline Bauer, Joachim Gerss. (2011). Longitudinal analysis of macronutrients and minerals in human milk produced by mothers of preterm infants. Clinical Nutrition. 30: 215-20.

[7] Moltó-Puigmartí C, Castellote AI, Carbonell-Estrany X, LópezSabater MC. (2011). Differences in fat content and fatty acid proportions among colostrum, transitional, and mature milk from women delivering very preterm, preterm, and term infants. Clinical Nutrition. 30:116-123.

[8] Hongliang W, Zhimin D. (2007). The effect of high artificial compound emulsion kidney load on milk infant kidney function. Laboratory Medicine and Clinic. 4:377 [article in chinese].

[9] Yazaki T, Umeyama T, Kaneko S, Kiriyama I, Ishikawa H, Koiso K. (1991). Multielement analysis of kidney tissue with renal calculi. Urology. 38: 290-3.

[10] Valentini RP, Lakshmanan Y. (2011). Nephrolithiasis in children. Adv Chronic Kidney Dis. 18:370-5. 